\title{
Evaluation of LIDAR and Camera External Reference Calibration Methods
}

\author{
Yao Fu, ${ }^{1}$ Dean Luo, ${ }^{1}$ He Huang, ${ }^{1}$ Yizhou Xue, ${ }^{1}$ and Tong Yin $^{2 *}$ \\ ${ }^{1}$ School of Geomatics and Urban Spatial Information, Beijing University of Civil Engineering and Architecture, \\ No. 15, Yongyuan Road, Huangcun Town, Daxing District, Beijing 102616, China \\ ${ }^{2}$ Institute of Surveying and Mapping Standardization, Ministry of Natural Resources, \\ 334 Youyi East Road, Xi'an, Shaanxi Province 710054, China
}

(Received July 21, 2021; accepted October 22, 2021; online published November 18, 2021)

Keywords: camera calibration, joint calibration, camera, LIDAR

In the implementation of autonomous driving, high-precision maps and environment perception are required to support the driving process. They are commonly used to fuse image and point cloud data, but it is necessary to obtain the external parameters of the camera and radar when performing data fusion. However, the external parameters of the camera and radar can cause problems that can be solved by joint calibration. For fast, accurate acquisition of external parameters, a special three-plane calibration plate is designed to fit the spatial equations for each of three different planes passing through the initial point clouds in this study. The calibration plate is used to obtain the coordinates of feature points in the radar coordinate system through the spatial relationships and to extract the pixel coordinates of the feature points from the images to establish the corresponding equations. Finally, the least squares method is used to obtain the calibration parameters. The experimental results show that this method can obtain calibration results faster and more robustly than the traditional checkerboard grid calibration method.

\section{Introduction}

At present, most of the sensors used for autonomous vehicle perception are composed of light detection and ranging (LIDAR) and vision systems. The perception of the external environment is realized through data fusion of multiple sensors. The collection and construction of highprecision map data also require data fusion of cameras and LIDAR, and through the data fusion of these sensors, the characteristics of each of the sensors can be used to improve the reliability of environmental recognition. ${ }^{(1)}$

The joint calibration methods of cameras and LIDAR for autonomous driving can be divided into online and offline calibration methods. ${ }^{(2,3)}$ The commonly used offline calibration methods are mainly the tessellation grid method and the feature point matching method.

The feature point matching method uses a special graphical calibration plate to extract 3D points acquired by LIDAR and the corresponding 2D image points obtained by a camera to

*Corresponding author: e-mail: yintong0907@126.com https://doi.org/10.18494/SAM.2021.3561 
establish constraint equations and optimize the computer pose, which is essentially a perspective$n$-point (PNP) problem for computer positional estimation. Zhou and Deng ${ }^{(4)}$ proposed a new robust algorithm and introduced weights to represent the uncertainty of the unit normal vector in the tessellation plane to obtain more accurate external parameters for the combined quality evaluation of each pair of images and LIDAR scans. Park et al. ${ }^{(5)}$ designed a type of triangular calibration plate for multi-line LIDAR, where 3D coordinates were obtained by fitting the point cloud data of multi-line LIDAR, and the corresponding pixel coordinates were obtained by the corner point detection method. However, radars with lower threads were limited because of the small number of marked target edge points. Gong et al. ${ }^{(6)}$ proposed a calibration method for a triangular calibration plate, which was calibrated by the nonlinear least squares method and then iteratively optimized to refine the calibration parameters. Debattisti et al., ${ }^{(7)}$ Pereira et al., ${ }^{(8)}$ and Pusztai and Haider ${ }^{(9)}$ used triangular plates, spheres, and shape diversity, respectively, to distinguish targets in the sensor data and achieved good calibration results. Wang et al. ${ }^{(10)}$ proposed a corner point extraction method based on the reflection intensity of the point cloud, which improved the accuracy of fusion results of camera and LIDAR data. Chen et al. ${ }^{(11)}$ used a special calibration cube with point-line constraints to establish the parametric equations between the laser feature points and image edges and calibrated the parameters by collecting data from different angle cubes. Geiger et al. ${ }^{(12)}$ used the idea of classifying the calibration plate laser point cloud and eliminating the point sets with a significantly low number of 3D points to achieve alignment. Hand et al. ${ }^{(13)}$ and Li et al. ${ }^{(14)}$ used LIDAR data points lying on a straight line to calibrate constraint relations using isosceles triangles and folded flat panels as calibrators, respectively. Guindel et al. ${ }^{(15)}$ achieved the automatic calibration of LIDAR and a stereo camera by using simple calibrators.

In this study, a basic study on the feature point method and the tessellation grid method is conducted, and a triangular calibration plate is designed to obtain the pixel coordinates of the feature corner points in the image data. The 3D coordinates of the feature points are determined using the point cloud plane obtained after fitting to establish the constraint equations for solving the parameters; these parameters represent a combination matrix of the rotation translation matrices of the camera. The accuracy of the calibration results is verified by calibration plate verification experiments using the actual alignment effect of the calibration plate on the image.

\section{Principle of Calibration in Checkerboard Grid Method}

Assume that two spatial normals from the calibration plate to the origin of the camera coordinate system and to the origin of the LIDAR coordinate system are denoted as $N_{C}$ and $N_{L}$, respectively. Then, the value of the modulus $\left\|N_{C}\right\|$ of $N_{C}$ is equal to the distance between the camera origin and the plane of the calibration plate, and the value of the modulus $\left\|N_{L}\right\|$ of $N_{L}$ is equal to the distance between the LIDAR origin and the plane of the calibration plate. The normal vectors $N_{C}$ and $N_{L}$ can be calculated from the available data.

Converting the rotation matrix $R$ from the world coordinate system (assuming the upper left corner of the board as the origin) to the camera coordinate system by Zhang's calibration method $^{(16)}$ using the translation vector $T$ yields 


$$
N_{C}=R_{3}\left(R_{3}^{T} t\right)
$$

where $R_{3}$ denotes the third vector of the rotation matrix $R$.

Then, the Point Cloud Library (PCL), which is an open-source programming library, is used in the relevant algorithm to obtain the overall point cloud in the calibration plate point cloud, and the least squares method or a random sampling consistency algorithm is used to fit the calibration plate plane. The equation of the fitted calibration plate plane equation is $A x+B y+C z+D=0$ and $(A, B, C)$ is the normal vector of the plane and also the unit vector. Then, $N_{L}$ can be expressed as

$$
N_{L}=[A, B, C]^{T} \sqrt{D}
$$

After obtaining the normal vectors $N_{C}$ and $N_{L}$, they are both linearly related to the rotation matrix $R_{L t o C}$ from the point cloud coordinate system to the camera coordinate system and the translation vector $T_{L t o C}$ from the point cloud coordinate system to the camera coordinate system. (1) The difference between $N_{L}$ and $N_{C}$, i.e., between the moduli $\left\|N_{L}\right\|$ and $\left\|N_{C}\right\|$, is equal to the length of the projection of the translation vector $T_{L t o C}$ on $N_{L}$, which can be expressed as

$$
\left\|N_{L}\right\|-\left\|N_{C}\right\|=T_{L t o C} n_{L}
$$

(2) Since $N_{L}$ and $N_{C}$ are both perpendicular to the calibration plate plane, they are parallel in the same coordinate system. To keep $N_{L}$ and $N_{C}$ in the same coordinate system, according to the principle of coordinate transformation, $n_{L}$ can be expressed as an $N_{C}$ unit vector of $R_{L t o C} n_{L}$, and $n_{C}$ in the camera coordinate system $O_{C}-X_{C}, Y_{C}, Z_{C}$ is expressed as $n_{C}=N_{C} /\left\|N_{C}\right\|$.

Since $R_{L t o C} n_{L}$ is parallel to $n_{C}$, the inner product of $R_{L t o C} n_{L}$ and $n_{C}$ is 1 .

$$
R_{L t o C} n_{L} n_{C}=1
$$

The above-defined $R_{L t o C}$ and $T_{L t o C}$ are used as initial solutions, and the objective function given by Eq. (5) is established. The initial solutions are optimized iteratively using the Levenberg-Marquardt (LM) nonlinear optimization algorithm.

$$
F=\sum_{i=1}^{n} \sum_{j=1}^{m}\left[\frac{N_{C, i}\left(R_{L t o C} P_{i, j}+T_{L t o C}\right)}{\left\|N_{C, i}\right\|}-\left\|N_{C, i}\right\|\right]^{2}
$$

In Eq. (5), $n$ is the number of positions in the data collected for the joint calibration, $m$ is the number of 3D laser point clouds scanned on the calibration plate at the $i$ th position, $N_{C, i}$ represents the normal 3D vector from the origin of the camera coordinate system to the plane of the calibration plate at the $i$ th position, $\left\|N_{C, i}\right\|$ is the modulus of the vector $N_{C, i}$, and $P_{i, j}$ represents the $j$ th laser scanning point on the calibration plate at the $i$ th position. 


\section{Camera and LIDAR Joint Calibration}

\subsection{Basic concept of joint calibration}

The geometric relationship between points in the 3D laser point cloud coordinate system and the 2D pixel coordinate system of a 2D plane is displayed in Fig. 1. The process of solving the model parameters represents the process of LIDAR and camera joint calibration, which can be transformed into an equation-solving problem. ${ }^{(17)}$

The basic principle of LIDAR and camera joint calibration is shown in Fig. 1. The LIDAR coordinate system $O_{L}-X_{L}, Y_{L}, Z_{L}$, camera coordinate system $O_{C}-X_{C}, Y_{C}, Z_{C}$, image coordinate system $O-X, Y$, and pixel coordinate system $O-u, v$ are set as shown in Fig. 1. There are linear relationships between the coordinate systems. Also, $\theta_{x}, \theta_{y}$, and $\theta_{z}$ denote the steering angles of the LIDAR coordinate system with respect to the camera coordinate system in the $x, y$, and $z$ directions, respectively, $T\left(t_{1}, t_{2}, t_{3}\right)$ is the translation vector from the camera coordinate system to the LIDAR coordinate system, and $\left(u_{0}, v_{0}\right)$ is the projection point of the image coordinate system origin to the pixel coordinate system. ${ }^{(18-20)}$ Figure 2 shows the flowchart of the feature point joint calibration method.

\subsection{Feature point joint calibration method}

The feature point method can obtain both 3D coordinates of a feature point in the LIDAR and 2D pixel coordinates of an image. To facilitate the acquisition of feature points, a special threeplane calibration plate is designed, as shown in Fig. 3. The calibration plate has three noncoplanar spatial planes denoted as planes 1,2 , and 3, and the three spatial points represent the three feature points to be obtained.

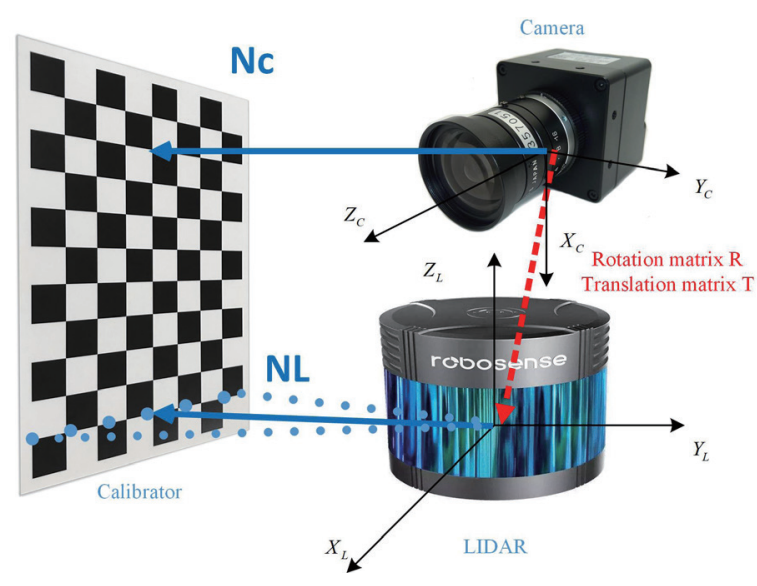

Fig. 1. (Color online) Schematic diagram of the checkerboard joint calibration method.

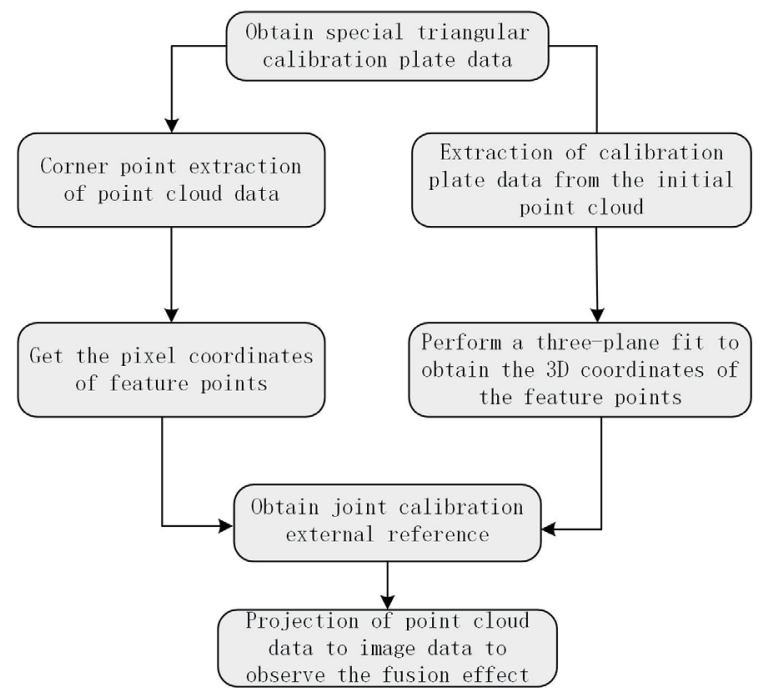

Fig. 2. Flowchart of the feature point joint calibration method. 


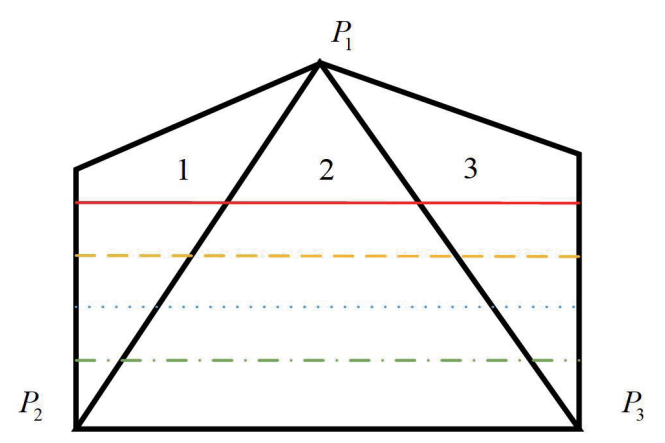

Fig. 3. (Color online) Special three-plane calibration board.

In the data acquisition process, to make planes 1, 2, and 3 obtain as many laser points as possible, a point cloud processing algorithm based on the points in the respective planes is used, and the face plane equation is derived as $A_{i} x+B_{i} y+C_{i} z+D_{i}=0$, where $i=1,2,3$; the corresponding plane normal to $V_{i}$ is $\left(A_{i}, B_{i}, C_{i}\right)(i=1,2,3)$. As shown in Fig. 3, the intersection of the three planes $P_{1}$ satisfies the following equations:

$$
\left\{\begin{array}{l}
A_{1} x+B_{1} y+C_{1} z+D_{1}=0 \\
A_{2} x+B_{2} y+C_{2} z+D_{2}=0 \\
A_{3} x+B_{3} y+C_{3} z+D_{3}=0
\end{array} .\right.
$$

The spatial coordinates of $P_{1}$ can be determined using the linear least squares method.

According to Fig. 3, the direction of vector $\overrightarrow{P_{1} P_{2}}$ should be the same as $V_{1} \times V_{2}$, and its length $\left|\overrightarrow{P_{1} P_{2}}\right|$ can be obtained from the calibration plate; then, vector $\overrightarrow{P_{1} P_{2}}$ is obtained. Similarly, we can obtain vector $\left|\overrightarrow{P_{1} P_{3}}\right|$.

$$
\begin{aligned}
& \overrightarrow{P_{1} P_{2}}= \pm \frac{V_{1} \times V_{2}}{\left|V_{1} \times V_{2}\right|}\left|\overrightarrow{P_{1} P_{2}}\right| \\
& \overrightarrow{P_{1} P_{3}}= \pm \frac{V_{3} \times V_{4}}{\left|V_{3} \times V_{4}\right|}\left|\overrightarrow{P_{1} P_{3}}\right|
\end{aligned}
$$

The values with the positive and negative signs in Eqs. (7) and (8) are combined with the calibration plate according to Fig. 3 . Since the coordinates of vectors $\overrightarrow{P_{1} P_{2}}, \overrightarrow{P_{1} P_{3}}$, and $P_{1}$ are known, the coordinates of points $P_{2}$ and $P_{3}$ can be calculated.

The 2D pixel coordinates of the feature points can be obtained by corner point detection with a manual check, and then the corresponding point sets of the feature points can be obtained. The calibration plate position is continuously changed for data acquisition, and three sets of feature points are obtained for each of the positions according to the above-presented method. The solution process after obtaining the feature points is as follows: 


$$
\begin{gathered}
Z_{c} u=n_{11} X_{L}+n_{12} Y_{L}+n_{13} Z_{L}+n_{14} \\
Z_{c} v=n_{21} X_{L}+n_{22} Y_{L}+n_{23} Z_{L}+n_{24} \\
Z_{c}=n_{31} X_{L}+n_{32} Y_{L}+n_{33} Z_{L}+n_{34}
\end{gathered}
$$

Substituting the third equation of Eq. (9) into the first two equations in Eq. (9) yields

$$
\begin{aligned}
& u\left(n_{31} X_{L}+n_{32} Y_{L}+n_{33} Z_{L}+n_{34}\right)=n_{11} X_{L}+n_{12} Y_{L}+n_{13} Z_{L}+n_{14} \\
& v\left(n_{31} X_{L}+n_{32} Y_{L}+n_{33} Z_{L}+n_{34}\right)=n_{21} X_{L}+n_{22} Y_{L}+n_{23} Z_{L}+n_{24}
\end{aligned} .
$$

Unlike the tessellation method, the eigenpoint method does not directly solve the internal reference and rotation matrix of the camera, but matrix $M$ in Eq. (10). After obtaining enough pairs of 3D LIDAR coordinates and 2D pixel coordinate point sets of the image, the constraint equations are established to solve matrix $M$. According to Eq. (10), two equations are established for a pair of point sets. Then, for $n$ sets of feature points, $2 n$ equations can be established as shown in Eq. (11):

$$
\left[\begin{array}{cccccccccccc}
x_{1} & y_{1} & z_{1} & 1 & 0 & 0 & 0 & 0 & -u_{1} x_{1} & -u_{1} y_{1} & -u_{1} z_{1} & -u_{1} \\
0 & 0 & 0 & 0 & x_{1} & y_{1} & z_{1} & 1 & -v_{1} x_{1} & -v_{1} y_{1} & -v_{1} z_{1} & -v_{1} \\
& & & & & \cdots & & & & & & \\
x_{n} & y_{n} & z_{n} & 1 & 0 & 0 & 0 & 0 & -u_{n} x_{n} & -u_{n} y_{n} & -u_{n} z_{n} & -u_{n} \\
0 & 0 & 0 & 0 & x_{n} & y_{n} & z_{n} & 1 & -v_{n} x_{n} & -v_{n} y_{n} & -v_{n} z_{n} & -v_{n}
\end{array}\right] A_{12 \times 1}=0
$$

Here, $A_{12 \times 1}=\left[n_{11}, n_{12}, n_{13}, n_{14}, n_{21}, n_{22}, n_{23}, n_{24}, n_{31}, n_{32}, n_{33}, n_{34}\right]$; if 12 parameters of $A_{12 \times 1}$ are required, a minimum of six sets of point pairs are needed. To improve the accuracy, usually more sets of points are used to increase the number of constraints, and then the linear least squares method is used to solve the problem.

\section{Experiments and Analysis of Results}

This study used an autonomous driving high-precision map acquisition platform developed by the authors for data acquisition to conduct joint calibration experiments and accuracy evaluation. The acquisition platform mainly included a Songling robot, a Hesai Pandar40 LIDAR, and a FLIR industrial camera, as shown in Figs. 4 and 5.

The Songling robot had the advantages of a long range, a high speed under this structure, a large weight capacity of up to $200 \mathrm{~kg}$, and support for the ROS system access. The LIDAR detection distance was $200 \mathrm{~m}$, having a $20 \%$ reflectivity; the vertical field of view was $23^{\circ}$, which provided a view range from -16 to $+7^{\circ}$; the minimum vertical angle resolution was $0.33^{\circ}$; the minimum horizontal angle resolution was $0.2^{\circ}$. The industrial camera sensor was a CCD having a resolution of $3376 \times 2704$ pixels and a display frame rate of 9 fps. 


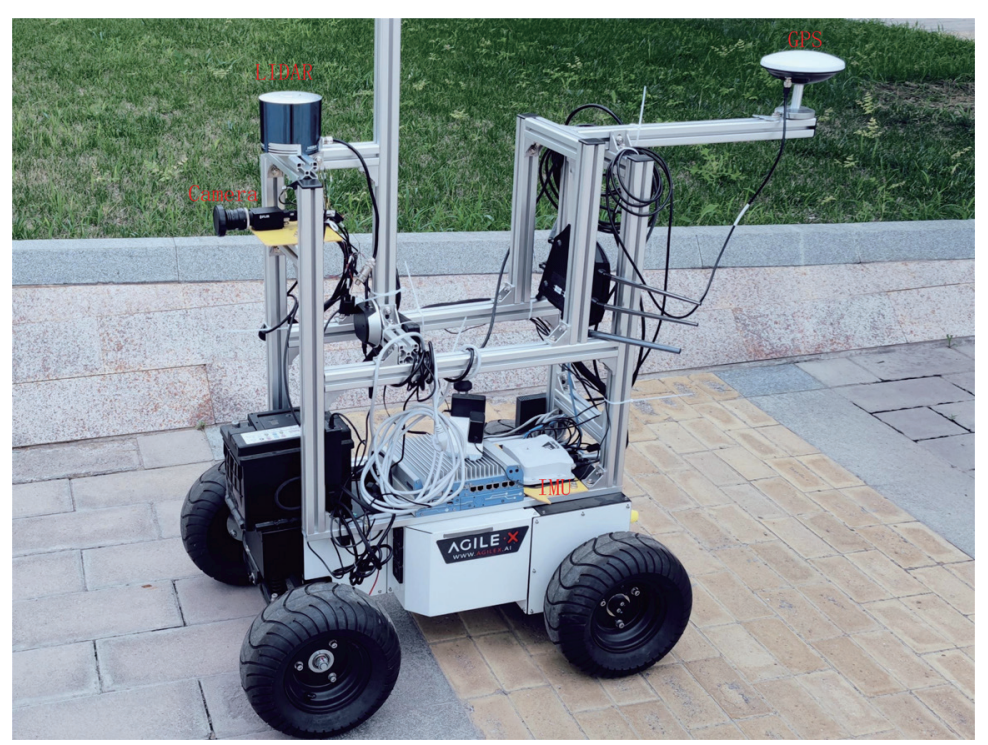

Fig. 4. (Color online) Autopilot equipment.

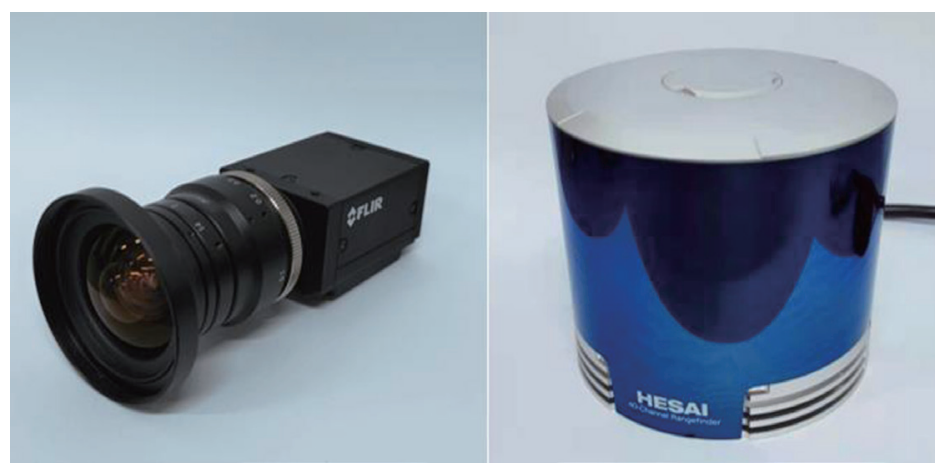

Fig. 5. (Color online) Camera and LIDAR.

\subsection{Checkerboard grid method experiment}

In accordance with the method described in Sect. 2.1, the checkerboard grid was placed in 16 different positions for the calibration of the LIDAR and the corresponding image feature points. First, the internal and external parameters of the camera were obtained by Zhang's calibration method. Then, the 3D vector was calculated by fitting the plane equation to the point cloud data of the camera calibration board and the initial solution of the rotation matrix of the point cloud coordinate system to the camera coordinate system. The translation vector from the point cloud coordinate system to the camera coordinate system was then calculated using the limiting equation. The LM nonlinear optimization algorithm was used to iteratively optimize the objective function of Eq. (5) to obtain the optimal solution and obtain the external reference matrix of the LIDAR and camera. 


\subsection{Joint calibration experiment of feature point method}

In the feature point method, corner point detection was first performed on the acquired images to obtain 2D coordinates of the pixel coordinate system. In this experiment, we used a special custom-built calibration plate for data acquisition, which was divided into two parts. The first part consisted of a lightweight tripod used to adjust the height of the calibration plate to facilitate the acquisition of point cloud data. The second part of the calibration plate had a similar shape to the board shown in Fig. 3, where planes 1 and 3 were right-angle triangles with sides of 60,80, and $100 \mathrm{~cm}$ and plane 2 was an isosceles triangle having a base length of $120 \mathrm{~cm}$ and two sides with a length of $100 \mathrm{~cm}$. When collecting the calibration plate point cloud data, the points on lines intersecting two planes were avoided, and the three planes were used to obtain as many point clouds as possible. The acquired image data are shown in Fig. 6.

In the experiment, the coordinates of feature points $P_{1}, P_{2}$, and $P_{3}$ were obtained by using corner point detection, but the result was not satisfactory, so a manual extraction method was used to obtain the coordinates of the corner points. The locations of the corner points of the feature points are shown as green points in Fig. 7.
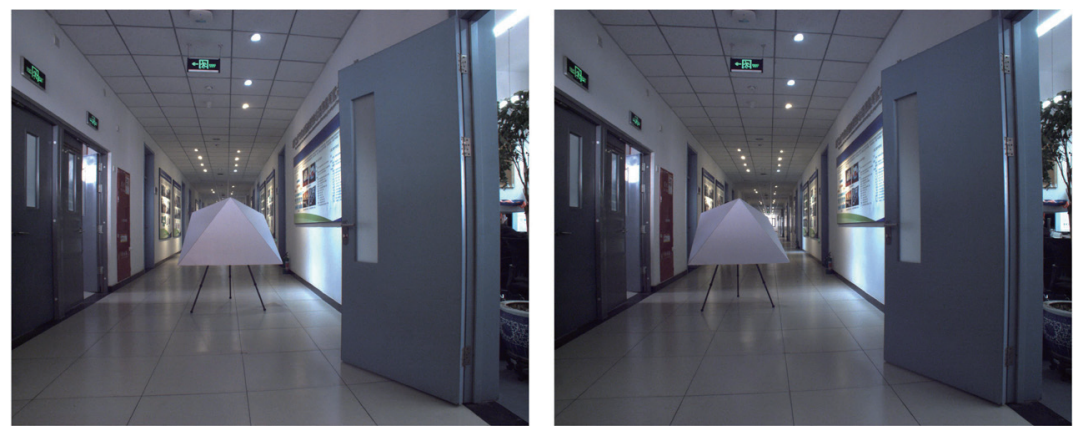

Fig. 6. (Color online) Image data.
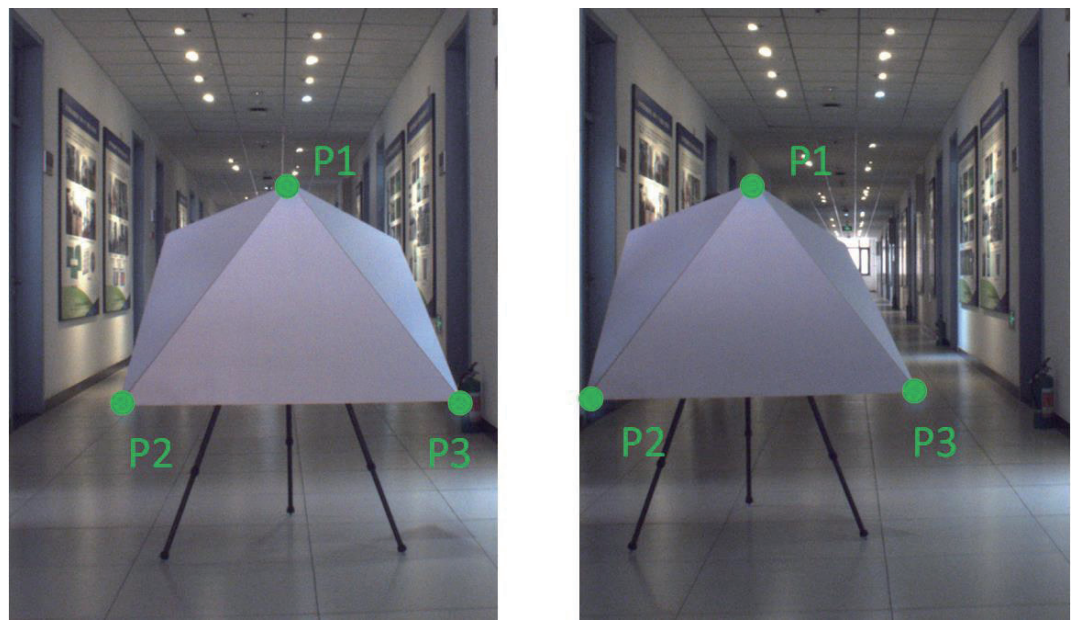

Fig. 7. (Color online) Corner point data acquisition. 
For the acquired point cloud data of the calibration plate, the least squares method was used to fit the plane to each point cloud of the calibration plate and to find the three-plane intersection point $\mathrm{P}_{1}$; then, the coordinates of points $P_{2}$ and $P_{3}$ were obtained. The point cloud image is shown in Fig. 8.

After obtaining the 3D coordinates of points $P_{1}, P_{2}$, and $P_{3}$ in the 3D laser coordinate system and the pixel coordinates in the pixel coordinate system, the over-constrained equations were solved using Eq. (11), whose solution contains 12 parameters, as shown in Table 1.

\subsection{Point cloud projection experiment}

To verify the calibration accuracy of the joint calibration method of feature points visually, the image and point cloud data of the calibration plate were collected in the south parking lot of an academic building in the Daxing campus of Beijing University of Architecture using a highprecision map acquisition platform. The results are shown in Fig. 9, where it can be seen that the point cloud of the calibration plate coincides well with the image data, and the point cloud of the trees on the left side coincides well with the point cloud of the vehicles in the rear, which demonstrates the feasibility of the feature point method.

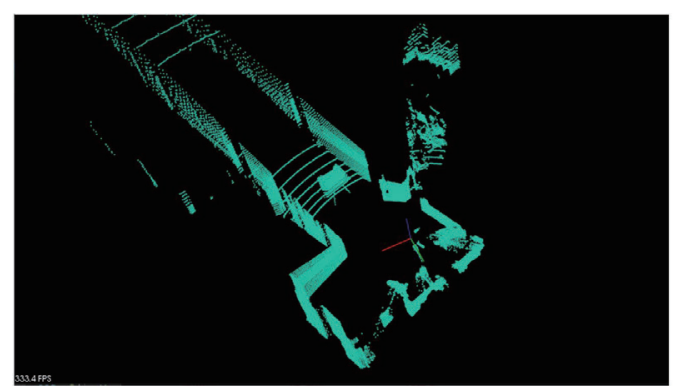

(a)

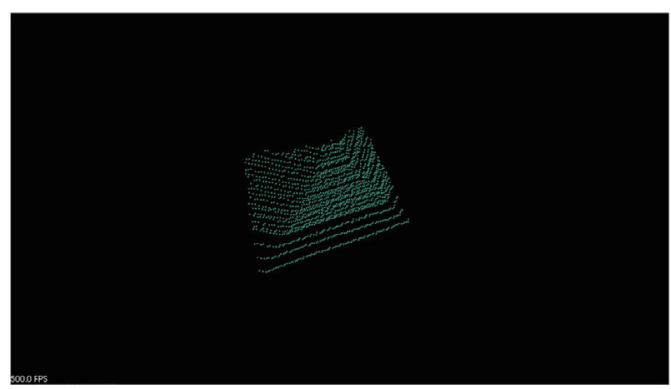

(b)

Fig. 8. (Color online) Point cloud image: (a) initial point cloud and (b) calibration board point cloud.

Table 1

Calibration result of the characteristic point method.

\begin{tabular}{lrrrr}
\hline $\begin{array}{l}\text { Experimental } \\
\text { sample group }\end{array}$ & \multicolumn{5}{c}{ Matrix parameters $A_{12 \times 1}$} \\
\hline \multirow{2}{*}{ Group 1 } & -1.00000 & -0.87567 & 0.27845 & -0.18630 \\
& -0.26866 & -0.67843 & -1.06084 & -0.31638 \\
& 0.00002 & -0.00049 & -0.00001 & -0.00006 \\
\hline \multirow{2}{*}{ Group 2 } & -1.00000 & -0.72830 & 0.25850 & -0.12702 \\
& -0.29605 & -0.61858 & -0.98411 & -0.28856 \\
& -0.00002 & -0.00046 & -0.00001 & -0.00006 \\
\hline \multirow{3}{*}{ Group 3 } & -1.00000 & -0.73259 & 0.25792 & -0.15787 \\
& -0.28786 & -0.63105 & -0.97909 & -0.37161 \\
& -0.00002 & -0.00046 & -0.00001 & -0.00006 \\
\hline
\end{tabular}




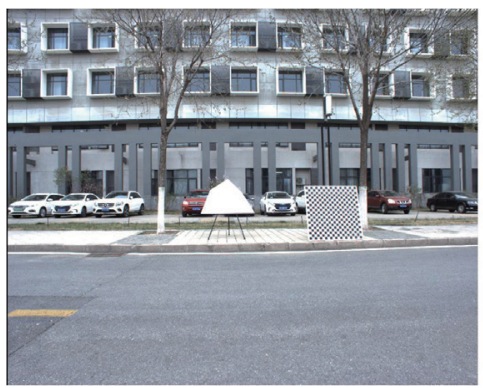

(a)

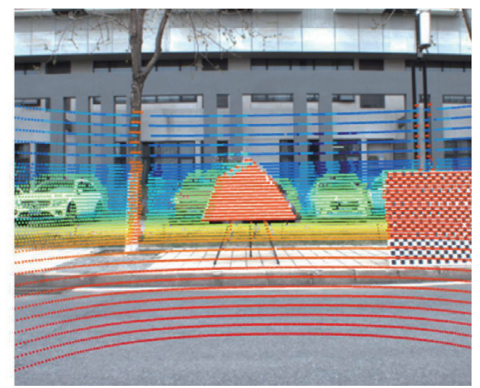

(b)

Fig. 9. (Color online) Point cloud projection results of calibration point method: (a) initial image data and (b) point cloud projection data of calibration board.

\subsection{Accuracy evaluation of compound origin coordinates}

In the accuracy verification of the algorithm, we considered that it may be difficult to fit the center of the point cloud using spherical targets and to obtain their two-bit pixel coordinates directly. Therefore, the specific targets used in the feature point method were used for the accuracy evaluation. The target was placed on the south side of an academic building in the Daxing campus of Beijing University of Architecture, so that its three planes could be scanned by the laser, and its $3 \mathrm{D}$ coordinates were obtained. The 2D pixel coordinates of the feature points were obtained from the image data.

The 3D coordinates of the target were linearly converted to the 2D pixel coordinates using the calibration parameters obtained by the tessellation grid method and the feature point method. The recovered pixel coordinates were compared with the true 2D pixel coordinates. The error was calculated by

$$
e=\sqrt{\left(u_{p, i}-u_{l, i}\right)^{2}+\left(v_{p, i}-v_{l, i}\right)^{2}},
$$

where $\left(u_{p, i}, v_{p, i}\right)$ denotes the true pixel point coordinates of the ith target and $\left(u_{l, i}, v_{l, i}\right)$ denotes the recovered pixel point coordinates of the $i$ th target. The error statistics are given in Tables 2 and 3.

The maximum, minimum, and average errors of the checkerboard grid method were 2.93, 1.51, and 2.12 pixels, whereas those of the characteristic point method were 4.73, 1.71, and 3.02 pixels, respectively.

In the feature point method, it was not necessary to perform camera calibration to obtain the camera's internal reference matrix; the rotation translation matrix was included in matrix $A$ in Eq. (11). Although the physical meaning of the internal reference matrix was not very intuitive, its calculation was relatively convenient, and the calculation used known feature points in the pixel coordinate system and radar coordinate system coordinates. In this study, the point cloud coordinates of the feature points were obtained by fitting the planes using a special calibration plate, whose pixel coordinates were obtained manually, which increased the error but made the calculation more efficient. 
Table 2

Error statistics of point coordinates recovered by checkerboard grid method. The unit is pixels.

\begin{tabular}{|c|c|c|c|c|c|c|}
\hline \multirow[t]{2}{*}{$\begin{array}{l}\text { Image } \\
\text { size }\end{array}$} & \multirow{2}{*}{$\begin{array}{c}\text { Test point } \\
\text { number }\end{array}$} & \multicolumn{2}{|c|}{$\begin{array}{c}\text { True 2D pixel } \\
\text { coordinates }(u, v)\end{array}$} & \multicolumn{2}{|c|}{$\begin{array}{l}\text { Pixel coordinates }(u, v) \text { obtained by } \\
\text { checkerboard grid method }\end{array}$} & \multirow{2}{*}{$\begin{array}{c}\text { Checkerboard method } \\
\text { projection results }\end{array}$} \\
\hline & & 1218 & 1216 & 1216.53 & 1213.96 & \\
\hline \multirow[t]{3}{*}{1} & 2 & 1017 & 1479 & 1015.42 & 1477.52 & 2.16 \\
\hline & 3 & 1428 & 1467 & 1426.32 & 1466.17 & 1.87 \\
\hline & 4 & 1439 & 1216 & 1438.28 & 1214.83 & 1.37 \\
\hline \multirow[t]{3}{*}{2} & 5 & 1242 & 1475 & 1240.12 & 1474.43 & 1.96 \\
\hline & 6 & 1646 & 1467 & 1645.01 & 1465.31 & 1.96 \\
\hline & 7 & 1606 & 1213 & 1604.28 & 1210.83 & 2.77 \\
\hline \multirow[t]{3}{*}{3} & 8 & 1408 & 1472 & 1410.12 & 1470.83 & 2.42 \\
\hline & 9 & 1811 & 1466 & 1810.32 & 1464.83 & 1.35 \\
\hline & 10 & 1746 & 1214 & 1743.98 & 1215.32 & 2.41 \\
\hline \multirow[t]{3}{*}{4} & 11 & 1558 & 1470 & 1556.32 & 1469.32 & 1.81 \\
\hline & 12 & 1952 & 1464 & 1950.39 & 1462.31 & 2.33 \\
\hline & 13 & 1901 & 1214 & 1903.12 & 1216.02 & 2.93 \\
\hline \multirow[t]{2}{*}{5} & 14 & 1703 & 1467 & 1705.23 & 1466.03 & 2.43 \\
\hline & 15 & 2100 & 1464 & 2101.21 & 1463.09 & 1.51 \\
\hline
\end{tabular}

Table 3

Error statistics of point coordinates recovered by characteristic point method. The unit is pixels.

\begin{tabular}{|c|c|c|c|c|c|c|}
\hline $\begin{array}{l}\text { Image } \\
\text { size }\end{array}$ & \multirow{2}{*}{$\begin{array}{c}\text { Test point } \\
\text { number }\end{array}$} & \multicolumn{2}{|c|}{$\begin{array}{c}\text { True 2D pixel } \\
\text { coordinates }(u, v)\end{array}$} & \multicolumn{2}{|c|}{$\begin{array}{l}\text { Pixel coordinates }(u, v) \text { obtained by } \\
\text { characteristic point method }\end{array}$} & \multirow{2}{*}{$\begin{array}{c}\text { Characteristic method } \\
\text { projection results }\end{array}$} \\
\hline & & 1218 & 1216 & 1215.32 & 1212.84 & \\
\hline \multirow[t]{3}{*}{1} & 2 & 1017 & 1479 & 1014.83 & 1476.29 & 3.47 \\
\hline & 3 & 1428 & 1467 & 1425.37 & 1464.75 & 3.46 \\
\hline & 4 & 1439 & 1216 & 1438.23 & 1214.47 & 1.71 \\
\hline \multirow[t]{3}{*}{2} & 5 & 1242 & 1475 & 1239.94 & 1474.76 & 2.07 \\
\hline & 6 & 1646 & 1467 & 1642.72 & 1463.59 & 4.73 \\
\hline & 7 & 1606 & 1213 & 1604.23 & 1210.89 & 2.75 \\
\hline \multirow[t]{3}{*}{3} & 8 & 1408 & 1472 & 1410.21 & 1469.34 & 3.46 \\
\hline & 9 & 1811 & 1466 & 1811.89 & 1468.79 & 2.93 \\
\hline & 10 & 1746 & 1214 & 1747.38 & 1216.17 & 2.57 \\
\hline \multirow[t]{3}{*}{4} & 11 & 1558 & 1470 & 1559.47 & 1467.69 & 2.74 \\
\hline & 12 & 1952 & 1464 & 1950.62 & 1466.52 & 2.87 \\
\hline & 13 & 1901 & 1214 & 1903.55 & 1216.42 & 3.52 \\
\hline \multirow[t]{2}{*}{5} & 14 & 1703 & 1467 & 1704.79 & 1466.62 & 1.83 \\
\hline & 15 & 2100 & 1464 & 2098.45 & 1461.32 & 3.10 \\
\hline \multicolumn{6}{|c|}{ Average value (unit: pixel) } & 3.02 \\
\hline
\end{tabular}

\section{Conclusion}

In this study, the calibration methods of LIDAR and camera sensors related to the sensing module in autonomous driving were investigated. In the joint calibration process, the calibration parameters obtained by the feature point calibration method were used for projection verification experiments and accuracy evaluation experiments, and the point cloud data and camera data were fused and used in the projection experiments. The average error of the recovered point 
coordinates compared with the true point coordinates was 3.02 pixels for the feature point method. To overcome the problems of the time-consuming and computationally demanding tessellation method, in the feature point method, camera calibration is not required to obtain the internal reference of the camera, and the rotation and translation matrices exist in a single matrix. Although the physical meaning of this matrix is not very intuitive, its calculation is relatively convenient and efficient, but the acquisition error of the feature point pixel coordinates is relatively large and needs to be improved. The experimental results show that both calibration methods have advantages and disadvantages, but they both have good practicality and high accuracy, and thus are suitable for use in actual applications.

\section{References}

1 F. Rodriguez, V. Fremont, and P. Bonnifait: IEEE Int. Conf. Multisensor Fusion and Integration for Intelligent Systems (2008) 214. https://doi.org/10.1109/MFI.2008.4648067

2 A. I. García-Moreno, J. J. González-Barbosa, A. Ramírez-Pedraza, J. B. Hurtado-Ramos, and F. J. OrnelasRodriguez: J. Appl. Remote Sens. 10 (2016) 024002. https://doi.org/10.1117/1.JRS.10.024002

3 Y. I. Abdel-Aziz, H. M. Karara, and M. Hauck: Photogramm. Eng. Remote Sens. 81 (2015) 103. https://doi. org/10.14358/PERS.81.2.103

4 L. Zhou and Z. Deng: Intell. Vehicles Symp. IEEE (2012) 642. https://doi.org/10.1109/IVS.2012.6232233

5 Y. Park, S. Yun, C. Won, K. Cho, K. Um, and S. Sim: Sensors 14 (2014) 5333. https://doi.org/10.3390/ $\underline{\mathbf{s} 140305333}$

6 X. Gong, Y. Lin, and J. Liu: Sensors 13 (2013) 1902. https://doi.org/10.3390/s130201902

7 S. Debattisti, L. Mazzei, and M. Panciroli: IEEE Intell. Vehicles Symp. (IV) (2013) 696. https://doi.org/10.1109/ IVS.2013.6629548

8 M. Pereira, D. Silva, V. Santos, and P. Dias: Robot. Auton. Syst. 83 (2016) 326. https://doi.org/10.1016/j. robot.2016.05.010

9 Z. Pusztai and L. Hajder: IEEE Int. Conf. Comput. Vision Workshops (ICCVW) 3 (2017) 94. https://doi. org/10.1109/ICCVW.2017.53

10 W. Wang, S. Ken, and K. Nobuo: Remote Sens. 9 (2017) 851. https://doi.org/10.3390/rs9080851

11 Z. Chen, L. Zhuo, K. Sun, and C. Zhang: Procedia Eng. 29 (2012) 4348. https://doi.org/10.1016/j. proeng.2012.01.669

12 A. Geiger, F. Moosmann, Ö. Car, and B. Schuster: Proc. IEEE Int. Conf. Robotics and Automation (2012) 3936. https://doi.org/10.1109/ICRA.2012.6224570

13 A. Hand, W. Friedl, M. Chalon, J. Reinecke, and M. Grebenstein: IEEE/RSJ Int. Conf. Intell. Robots Syst. (2011) 1366. https://doi.org/10.1109/IROS.2011.6048131

14 G. Li, Y. Liu, L. Dong, X. Cai, and D. Zhou: Int. Conf. Intell. Robots Syst. (2007) 3854. https://doi.org/10.1109/ ICRA40945.2020.9197316

15 C. Guindel, J. Beltrán, D. Martín, and F. García: IEEE Int. Conf. Intell. Transp. Syst. (2017). https://arxiv.org/ abs/1705.04085v1

16 Z. Zhang: 7th IEEE Int. Conf Computer Vision IEEE (1999) 666. https://doi.org/10.1109/ICCV.19 99.791289

17 Z. Huang, Y. Su, Z. Wang, and C. Zhang: Chin. J. Sci. Instrum. 41 (2020) 121. https://doi.org/10.19650/j.cnki. cjsi.J2006756

18 G. Kang, Q. Zhang, H. Zhang, W. Xu, and W. Zhang: Chin. J. Sci. Instrum. 40 (2019) 118. https://doi. org/10.19650/j.cnki.cjsi.J1905674

19 T. Tóth, Z. Pusztai, and L. Hajder: IEEE Int. Conf. Robotics and Automation (ICRA) (2020) 8580. https://doi. org/10.1109/ICRA40945.2020.9197316

20 R. Ishikawa, T. Oishi, and K. Ikeuchi: 2018 IEEE/RSJ Int. Conf. Intell. Robots Syst. (IROS) (2019). https:// arxiv.org/abs/1804.05178v1 


\section{About the Authors}

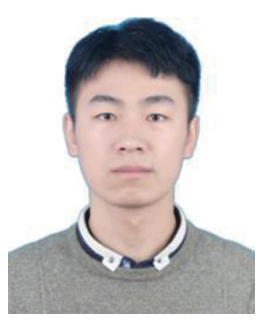

Yao Fu received his B.S. degree from Beijing University of Civil Engineering and Architecture, China, in 2020, where he is now studying for his M.S. degree. His research interests are in the joint calibration of cameras and LIDAR.

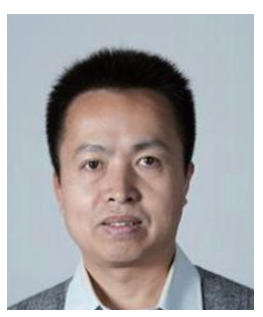

Dean Luo received his B.S. degree from Wuhan University, China, in 1990 and his M.S. and Ph.D. degrees from Southwest Jiaotong University, China, in 1997 and 2002, respectively. Since 2004, he has been a lecturer and professor at Beijing University of Civil Engineering and Architecture, China. His research interests are in GNSS, geodetic technology, and deformationmonitoring technology.

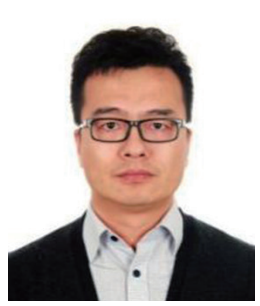

He Huang received his B.S. degree from Wuhan University, China, in 2000 and his M.S. and Ph.D. degrees from Sungkyunkwan University, South Korea, in 2004 and 2010, respectively. Since 2010, he has been a lecturer and associate professor at Beijing University of Civil Engineering and Architecture, China. His research interests are in autonomous driving, high-precision navigation maps, and visual navigation and positioning.

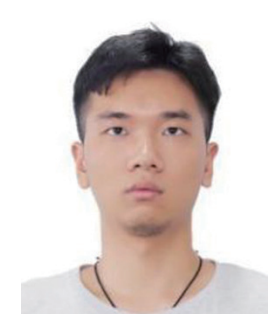

Yizhou Xue received his B.S. degree from Beijing University of Civil Engineering and Architecture, China, in 2018, and a master's degree in 2021. His research interests are in the joint calibration of cameras and LIDAR.

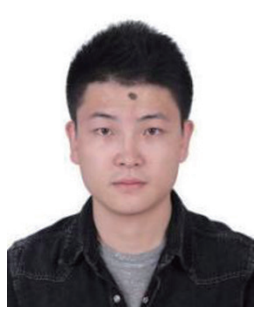

Tong Yin received his B.E. degree from Central South University, China, in 2012 and his M.S. degree from the University of Nottingham, United Kingdom, in 2015. From 2015 to 2017, he was an assistant engineer at the First Topographic Surveying Brigade of the Ministry of Natural Resources. Since then, he has been an engineer at the Institute of Surveying and Mapping Standardization of the Ministry of Natural Resources. His research interests are the application of positioning and navigation technology and the standardization of surveying, mapping, and geo-information.

(yintong0907@126.com) 